Ann. Zootech., I968, 17 (2), I69-I82.

\title{
MESURE QUANTITATIVE \\ DE LA PRODUCTION LAITIÈRE CHEZ LA LAPINE
}

\author{
I. I.EBAS \\ aver: la collaboration technique de G. SARD1 \\ Station de Recherches sur l'Elevage des Porcs, \\ Centre national de Recherches zootechniques, 78 -Jouy-en-Josas \\ Institut national de la Recherche agronomique
}

SOMMAIRE

Ce travail consiste en la mise au point d'une technique de mesure des quantités de lait produites par la lapine au cours de toute la durée de l'allaitement. Ia méthode proposée utilise l'estimation de la perte de poids de la lapine au cours de la tétée, un jour sur deux. Cette évaluation comporte une erreur maximum de 7,3 p. Ioo par rapport it la production réelle.

La production laitiere moyenne ( 143 lactations) pour 6 semaines est de $7,09 \pm 0,28 \mathrm{~kg}$ pour la race Fauve de Bourgogne. Ia courbe de lactation moyeme est asymétrique et présente un maximum aux environs du $19^{\mathrm{e}}$ jour. Les relations existant entre un certain nombre de fractions de la production laitičre et la production totale sont étudiées ensuite en vue d'une estimation de la production totale par une de ses parties. Ces estimations sont comparces à celles obtenues en pratiquant des contrôles plus ou moins espacés de la production laitière.

\section{IN'TRODUC'TION}

La production laitière de la lapine conditionne la survie et la croissance des lapereaux; en effet, les jeunes animaux naissent aveugles et sont à peine capables de se déplacer. Ce n'est que tardivement qu'ils commencent à consommer un aliment autre que le lait maternel. Les travaux relatifs à cette période importante de la vie de la lapine reproductrice sont peu nombreux, souvent imprécis, mais surtout incomplets pour la période considérée : de la mise bas au sevrage.

Nous nous sommes donc attachés à mettre au point une méthode de mesure des quantités de lait produites par la lapine, pouvant s'appliquer durant toute la période d'allaitement. L'exposé de cette méthode et les premiers résultats font l'objet de la présente étude.

Annales de Zootechnie. - I968. 


\section{MATÉRIEI, ET MÉTHODES}

\section{A. - Les animaux}

Les 6r lapines utilisées pour cette étude sont de race Fauve de Bourgogne. Tous les animaux sont élevés dans des cages métalliques à sol grillagé, installées dans un bâtiment fermé de construction légère et à ventilation statique. Un chauffage à air pulsé permet de maintenir la température audessus de $12^{\circ} \mathrm{C}$ en période hivernale. L'humidité relative à l'intérieur du bâtiment est en moyenne de $70 \mathrm{p}$. roo, mais elle peut varier de $40 \mathrm{p}$. 100 à $100 \mathrm{p}$. 100 selon les conditions atmosphériques.

Chaque lapine et sa portée sont logées dans deux cages contiguës et séparées. Les lapereaux sont mis en présence de la mère une seule fois par jour pour une tétée surveillée. Le sevrage est effectué 6 semaines après la mise bas. La mère et les petits disposent en permanence d'eau propre (distribution automatique) et du même aliment composé complet présenté en granulés de $2,5 \mathrm{~mm}$ de diamètre et de $8 \mathrm{~mm}$ de longueur environ. La composition de cet aliment est donnée au tableau I.

TABLEAU I

Composition de l'aliment distribué en cours de lactation

\begin{tabular}{|c|c|c|c|}
\hline Éléments constitutifs & P. 100 & Composition chimique & $\begin{array}{l}\text { P. } 100 \text { de la } \\
\text { matière frâiche }\end{array}$ \\
\hline 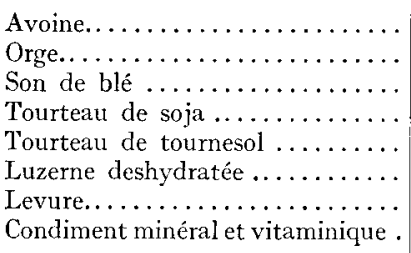 & $\begin{array}{r}20 \\
15 \\
10 \\
20 \\
9 \\
20 \\
2 \\
4\end{array}$ & 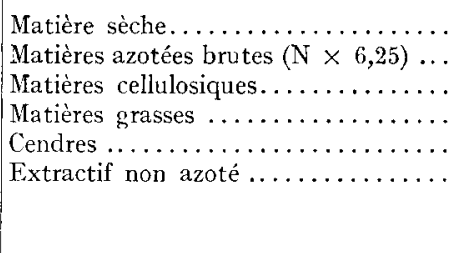 & $\begin{array}{r}90,7 \\
23,1 \\
10,6 \\
2,2 \\
9,3 \\
45,5\end{array}$ \\
\hline
\end{tabular}

B. - Choix de la méthode de mesure

A priori, plusieurs méthodes sont possibles pour mesurer la sécrétion lactée chez la lapine. II s'agit, soit de mesure directe par traite de la lapine, soit de mesure indirecte par estimation des variations de poids des animaux au cours de la tétée.

\section{Mesure directe par traite de la lapine.}

Il s'agit d'appliquer aux lapines la technique utilisée pour les vaches, les brebis : une traite mécanique ou manuelle de la mamelle. Cette technique exige de fortes quantités d'ocytocine $(2$ à 3 UI par animal) qui sont roo fois supérieures à celles libérées naturellement au cours d'une tétée. Les quantités de lait ainsi obtenues (Io à $80 \mathrm{~g}$ ) sont faibles et ne représentent qu'un quart à un dixième du lait effectivement présent dans la mamelle (DAvies et al., ig64; CoATEs et al., ig64). Pour ces différentes raisons, la méthode n'a pu être retenue pour notre étude.

\section{Mesure indirecte par les variations de poids au cours de la tétée.}

La mesure de la perte de poids de la lapine ou du gain de poids de la portée au cours de la tétée fournit une estimation de la quantité de lait ingérée par les lapereaux. La somme de ces mesures au cours d'une période équivaut sensiblement à la production laitière de la mère. Afin de déterminer s'il convient de peser la mère ou sa portée, 25 lactations ont été contrôlées par les deux méthodes simultanément. Les mères et les portées ont été pesées avant et après chaque tétée, tous les jours durant 6 semaines. 


\section{C. - Technique de mesure, fréquence des contrôles}

Indépendamment de la méthode utilisée pour mesurer la quantité de lait relative à une tétée déterminée, se pose le problème de la fréquence à adopter dans le contrôle des tétées par rapport à la fréquence naturelle de ces dernières.

Spontanément, la lapine n'allaite ses petits qu'une seule fois par 24 heures. Cette particularité de l'espèce a déjà été observée par de nombreux auteurs (Cross et HarRIS, 1952; Davis, 1957 ; VENGE, I963; DAVIES et al., I964; ZARROW et al., I965) aussi, la considérons-nous comme constante.

La précision obtenue dans l'estimation de la production laitière totale avec des contrôles à fréquence variable a été calculée à partir de la production laitière de 35 lapines, mesurée par un contrôle quotidien des tétées. Grâce à ces données, nous avons déterminé la production laitière totale estimée lors de contrôles à périodicité variable. Pour ce calcul, nous avons évalué les données théoriquement manquantes par la moyenne de celles qui les encadrent.

\section{D. - Technique de pesée}

Toutes les pesées ont été effectuées sur une balance automatique de ro kg de portée, permettant de réaliser des mesures avec une précision de $\pm \mathrm{I}$ g. Le même plateau permet de recevoir une lapine ou sa portée. Toutefois, quand la portée dépassait 9 à $10 \mathrm{~kg}$, les pesées étaient effectuées en 2 fois.

\section{RÉSULTATS ET DISCUSSION}

A. - Étude critique de la méthode de mesure

\section{Faut-il peser la lapine ou sa portée?}

Les résultats moyens obtenus par pesée de la mère et de la portée avant et après chaque tétée figurent au tableau 2.

\section{TABLEAU 2}

Productions laitières moyennes obtenues selon deux méthodes de pesée (25 lactations)

\begin{tabular}{|c|c|c|c|c|c|c|c|c|}
\hline Semaines & 1 & 2 & 3 & ' & 5 & 6 & Total & $\frac{A-B}{A} \times 100$ \\
\hline $\begin{array}{l}\text { Lait mesuré par pesée de la } \\
\text { mère (moyenne en grammes) }\end{array}$ & $\begin{array}{r}705 \\
\pm \quad 110\end{array}$ & $\begin{array}{r}1152 \\
\pm \quad 180\end{array}$ & $\begin{array}{r}1290 \\
+220\end{array}$ & $\begin{array}{r}1169 \\
+200\end{array}$ & $\begin{array}{r}915 \\
+\quad 150\end{array}$ & $\begin{array}{r}692 \\
+130\end{array}$ & $\begin{array}{l}A_{5} 912 \\
\pm 910\end{array}$ & \\
\hline $\begin{array}{l}\text { Lait mesuré par pesée de la } \\
\text { portée (moyenne en grammes) }\end{array}$ & $\begin{array}{r}643 \\
\pm \quad 100\end{array}$ & $\begin{array}{r}1086 \\
\pm 170\end{array}$ & $\begin{array}{r}1226 \\
\pm 210\end{array}$ & $\begin{array}{r}1117 \\
\pm 190\end{array}$ & $\begin{array}{r}869 \\
\pm 110\end{array}$ & $\begin{array}{r}626 \\
\pm 120\end{array}$ & $\begin{array}{l}\text { B5 } 548 \\
\pm 850\end{array}$ & \\
\hline
\end{tabular}

* Différence hautement significative (méthode des couples) $(\mathrm{P}<0,01)$. 
La différence entre les deux méthodes est relativement importante et hautement significative. L'écart observé peut en partie s'expliquer par les mictions non contrôlables des lapereaux ( $\mathrm{O}-\mathrm{I} 5 \mathrm{~g})$ et par la perte due à la respiration de ceux-ci (2 g maximum).

L'agitation des lapereaux principalement avant la tétée limite la précision de la mesure. Les pesées ne peuvent être faites avec une approximation supérieure à $\pm 6 \mathrm{~g}$ avant la tétée et $\pm 4 \mathrm{~g}$ après. La quantité de lait est ainsi estimée avec une erreur de \pm Io $\mathrm{g}$. Par contre, le calme des lapines permet une lecture à $\pm 2 \mathrm{~g}$ pour chacune des pesées, soit une erreur maximum de $\pm 4 \mathrm{~g}$ sur la quantité de lait estimée par pesée de la mère.

Pour ces différentes raisons (mictions, respiration, précision...) il nous a semblé préférable d'estimer la production laitière par la perte de poids de la lapine au cours de la tétée plutôt que par le gain de poids de la portée.

\section{Fréquence des contrôles.}

A partir des 35 lactations, mesurées par un contrôle quotidien des tétées sur la mère durant 6 semaines, il est possible de calculer la précision des estimations de production laitière faites en n'utilisant que des mesures espacées. Les calculs ont été effectués pour les fréquences suivantes : I jour sur 7 en commençant le I er et en terminant le $42^{e}$ jour, I jour sur 2, 5 jours consécutifs sur 7 , et enfin, 6 jours sur 7 . Les résultats de ce calcul théorique figurent au tableau 3 .

TABIEAU 3

Comparaison de 1 méthodes d'estimation de la production laitière

$$
(n=35)
$$

\begin{tabular}{|c|c|c|c|}
\hline Type rle contrôle & $\begin{array}{c}\text { Quantité de lait } \\
\text { moyenne par jour } \\
\text { (n) }\end{array}$ & $\begin{array}{c}\text { Erreur maxi- } \\
\text { 'mum }\left(I^{\prime}===0,05\right) \\
(\% / 0)\end{array}$ & $\begin{array}{l}\text { Sinnitication } \\
\text { de l'erreur }\end{array}$ \\
\hline Tous les jours .... & $\operatorname{lix}, 8$ & $!$ & \\
\hline 6 jours sur $7 \ldots \ldots$ & 169,0 & 2,5 & $\times S$ \\
\hline 5 jours sur $7 \ldots \ldots$ & $\operatorname{lng} .: 3$ & $\pm \quad 3,8$ & $X S$ \\
\hline 1 jour sur $2 \ldots$. & llis', & $\pm 1,8$ & $\therefore$ \\
\hline 1 jour sur $7 \ldots$ & 117,2 & $\pm 15,0$ & NS \\
\hline
\end{tabular}

Les trois premières méthodes donnent des résultats comparables. La précision augmente avec la fréquence des mesures. Le contrôle un jour par semaine, par contre, introduit une erreur beaucoup plus importante diminuant très nettement la précision dans l'estimation. Pour des raisons de main-d'œuvre et d'organisation du travail, nous avons retenu, dans la pratique, la mesure un jour sur deux. Les jours sans contrôle, la mère est mise en présence des petits dans les mêmes conditions, pour une tétée qui dure 3 à 5 minutes. La quantité de lait tétée est estimée par la moyenne de celles des deux jours qui l'encadrent. 
3. Précision dans l'estimation de la production laitière.

Plusieurs causes peuvent venir diminuer la précision des mesures réalisées à savoir :

- la perte de poids des lapines par respiration : elle n'est pas mesurable entre les 2 pesées nécessitées par le contrôle, et dont l'écart maximum est de 7 à 8 minutes. Ellle a été considérée comme nulle contrairement à celle de la portée pour la même durée;

- les pertes par déjections : contrairement aux lapereaux, les lapines adultes n'urinent ni ne défèquent jamais au cours des manipulations ou lors de la tétée. Ces pertes sont nulles;

- la quantité de lait s'estime à \pm 4 g près, soit une erreur relative de $2,5 \mathrm{p}$. Ioo, pour une production laitière moyenne de $I 69 \mathrm{~g}$ par jour;

- l'estimation de la production laitière par mesure d'une tétée sur deux se fait avec une précision de $\pm 4,8$ p. Ioo (tabl. 3).

La production laitière d'une lapine peut donc être estimée, avec une erreur maximum de 7,3 p. Ioo dans le cas d'un contrôle tous les 2 jours par mesure de la perte de poids de la femelle. Flle ne serait que de $\pm 5,0 \mathrm{p}$. Ioo pour 6 tétées contrôlées sur 7 .

La comparaison de la précision de notre méthode avec celle d'autres auteurs n'étant pas possible dans le cas de la Lapine, il est intéressant de la confronter avec des méthodes analogues utilisées chez d'autres femelles domestiques. A cet égard, SALMON-LEGAGNEUR (I956) observe qu'un contrôle hebdomadaire de 6 heures de la production laitière de la Truie entraîne une erreur maximum de \pm I 8 p. Ioo au seuil de probabilité $\mathrm{P}=0,05$ par rapport à un enregistrement de la totalité des tétées. La précision est semblable à celle que nous avons observée avec des mesures un jour par semaine ( \pm I 7,5 p. Ioo d'erreur maximum). I)ans les mêmes conditions, RICoRDEAU et al. (I960) estiment pour la Brebis l'erreur faite, à un niveau un peu plus faible : \pm I2 à I4 p. Ioo maximum.

Pour la Vache, en tenant compte des écarts réels entre des contrôles mensuels, CARRÉ et al. (I959) estiment que l'erreur faite dans l'estimation de la production laitière est au maximum de $\pm 7,4 \mathrm{p}$. Ioo de la production réelle $(\mathrm{P}=0,05)$, compte non tenu des erreurs de pesée. Il faut cependant souligner qu'il s'agit pour la Vache de production laitière mesurée par traite directe et non pas une méthode indirecte comme dans nos autres espèces. Pour la Lapine, un contrôle tous les deux jours, beaucoup plus astreignant, permet tout juste d'avoir une précision supérieure $( \pm 4,8 \mathrm{p}$. IOo).

\section{B. - La lactation de la Lapine}

Cent quarante-trois portées nées de mars I 966 à mars I 967 , ont été suivies pour le contrôle de lactation. Elles provenaient de 6I lapines, soit en moyenne 2,3 portées contrôlées par mère.

\section{Production laitière moyenne.}

La production laitière totale au cours de la lactation (42 jours) a été en moyenne de $7,09 \pm 0,28 \mathrm{~kg}$. La distribution des lactations est rapportée à la figure $\mathbf{r}$. Le tableau 4 indique quelques paramètres caractéristiques de ces lactations. 
TABLEAU 4

Quelques paramètres relatifs aux 143 lactations

\begin{tabular}{|c|c|c|}
\hline Caractéristiques moyennes & Mise bas & Sevrage \\
\hline Nombre de lapereaux par portée & $9,08 \pm 0,36$ & $8,22 \pm$ \\
\hline Poids de portée (en grammes) & $478 \pm 19$ & $8220 \pm 120$ \\
\hline Poids des lapines (en grammes) & 4301 I 83 & $4633 \pm 95$ \\
\hline
\end{tabular}
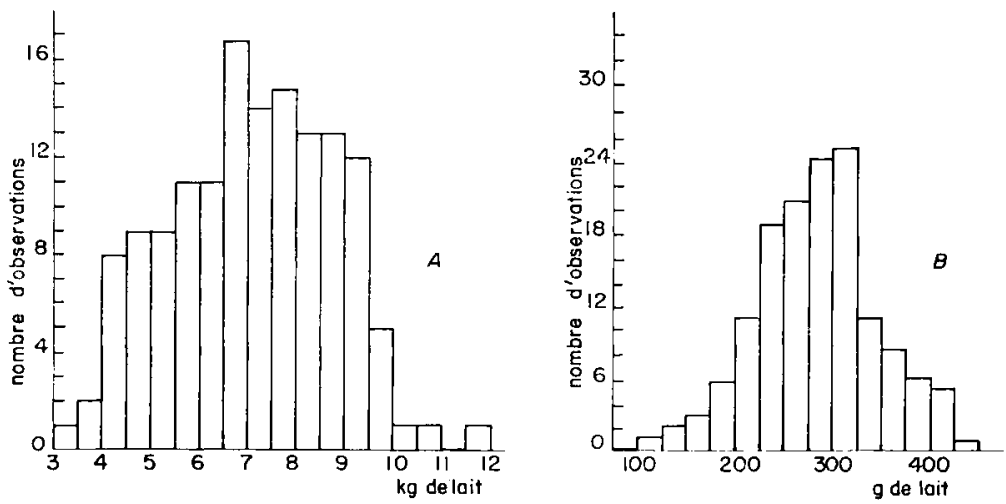

FIG. I. - Distribution des productions laitieres des lapines $(n=\mathrm{I}+3)$
A. - Production laitière totale
B. - Maximum journalier

A titre indicatif, quelques productions laitières moyennes ont été relevées chez différents auteurs. Ces résultats moyens quotidiens figurent au tableau 5 en comparaison de celui relevé par nos soins.

\section{TABLEAU 5}

Production laitière moyenne quotidienne selon quelques auteurs

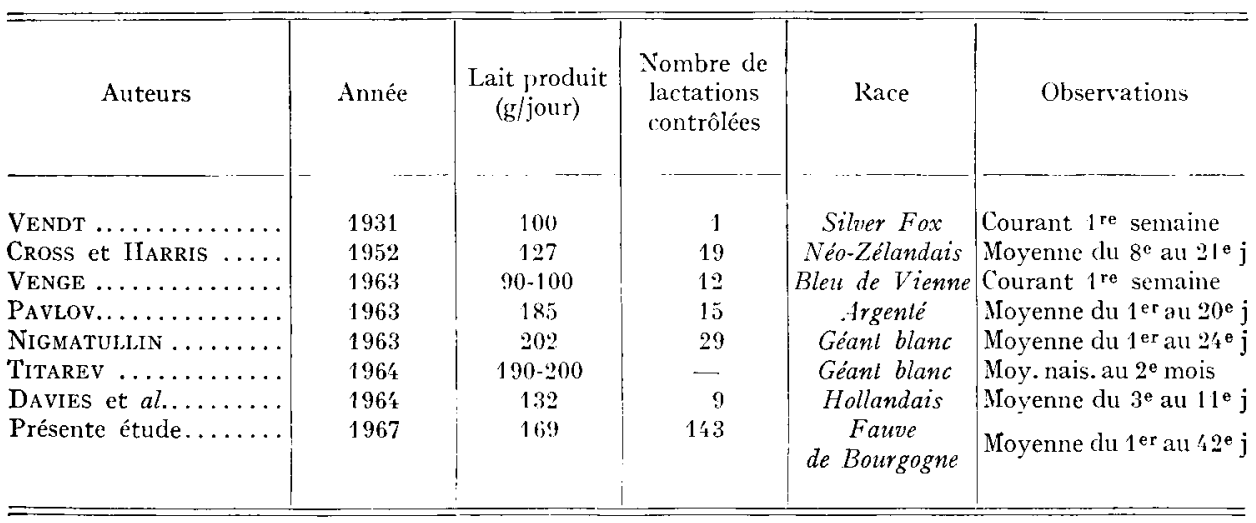


L'analyse de ce tableau indique une relative homogénéité dans les résultats des différents auteurs. Les variations existantes peuvent s'expliquer par le format des différentes races utilisées, ainsi que par le stade auquel les lactations ont été contrôlées.

\section{2. Évolution dans le temps.}

L'évolution hebdomadaire des quantités de lait produites ainsi que le nombre de lapereaux, sont rapportés au tableau 6.

\section{TABLEAU 6}

Évolution hebdomadaire de la production laitière et des effectifs de portée $\left(n=x_{43}\right)$

\begin{tabular}{|c|c|c|c|c|c|c|c|}
\hline Semaines & 1 & 9 & 3 & 4 & 5 & 6 & de 0 à 42 jours \\
\hline Production laitière (en g) & $\begin{array}{r}812 \\
\pm 37\end{array}$ & $\begin{array}{r}1291 \\
\pm 60\end{array}$ & $\begin{array}{r}1690 \\
\pm 68\end{array}$ & $\begin{array}{r}1460 \\
\pm 63\end{array}$ & $\begin{array}{l}1030 \\
\pm 56\end{array}$ & $\begin{array}{r}680 \\
\pm 49\end{array}$ & $\begin{array}{r}7093 \\
\pm 280\end{array}$ \\
\hline $\begin{array}{c}\text { Nombre de lapereaux } \\
\text { par portée }\left(^{(1)}\right.\end{array}$ & 8,47 & 8,32 & 8,31 & 8,25 & 8,23 & 8,22 & 8,3 \\
\hline
\end{tabular}

$\left({ }^{1}\right) \pm 0,33$

Il est à remarquer que le maximum de production situé à la $3^{\mathbf{e}}$ semaine, représente deux fois la quantité produite en première semaine et plus de deux fois celle produite au cours de la $6^{\mathrm{e}}$ semaine. On trouvera, sur la figure 2 , la répartition des maxima de chacune des lactations en fonction de la date à laquelle ils ont a été observés.

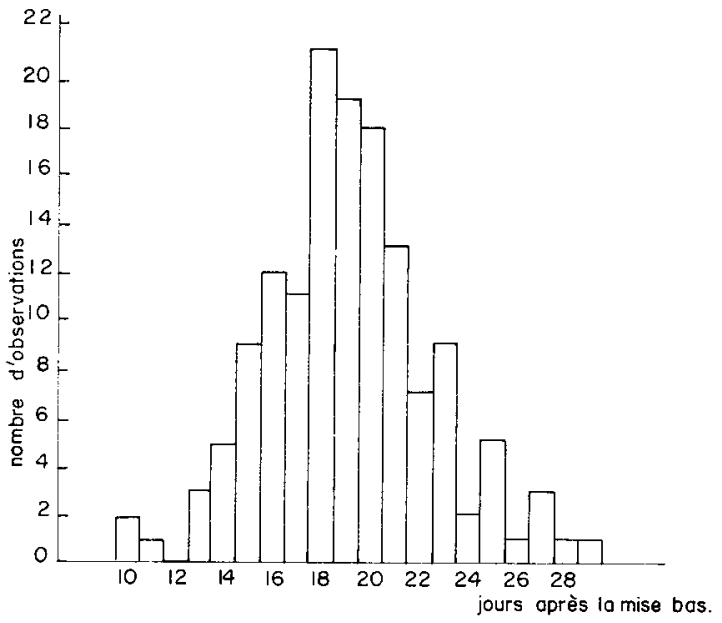

FIG. 2. - Date du maximum journalier de production laitière $(n=143)$ 
Le point maximum des courbes individuelles de production laitière est situé dans le courant de la $3^{\mathrm{e}}$ semaine pour $72 \mathrm{p}$. Ioo des lactations contrôlées. Il est intéressant de remarquer que c'est précisément à cette époque que les lapereaux commencent à consommer un aliment complémentaire, aussi nous proposons-nous de rechercher au cours d'une prochaine étude, l'existence d'une éventuelle relation entre la date d'apparition du maximum et le début de consommation d'aliments solides par les lapereaux.

La courbe de lactation présentée à la figure 3 a été établie à partir de la mestre de 3784 tétées. A titre indicatif on trouvera sur les figures 4 et 5 deux exemples de

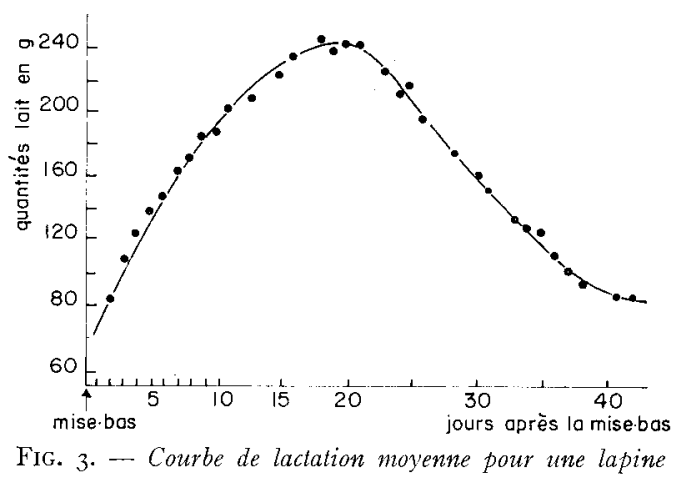

courbes de lactation individuelles. Elles indiquent quelles peuvent être les variations enregistrées dans la production d'un jour sur l'autre. Toutes deux présentent la même forme générale que la courbe moyenne avec deux phases de courbure inverse. Toutefois, l'importance des deux phases peut varier d'une lactation à l'autre comme 1'indique la figure 6 . Les 2 courbes sont obtenues en utilisant les résultats hebdomadaires de deux lapines ayant produit presque la même quantité totale de lait en 42 jours : $8,07 \mathrm{~kg}$ pour la lapine I Io et $8,04 \mathrm{~kg}$ pour la I07. Elles étaient respectivement à leur $3^{\mathrm{e}}$ et $2^{\mathrm{e}}$ portées.

L'observation des courbes de lactation individuelles sotuligne les variations de production pouvant se produire d'un jour à l'autre : 50 à Ioo p. IOO. L'une des courbes est qualifiée de régulière, l'autre d'irrégulière, mais tous les types intermédiaires existent.

La forme générale des courbes individuelles est celle de la courbe moyenne dans la grande majorité des cas; cependant, pour quelques lapines mauvaises laitières, elle présente des courbures inversées.

Sauf exceptions dues à des cas fortuits, la courbe de lactation des lapines ne présente jamais qu'un seul maximum, contrairement aux observations de SALMONLEGAGNEUR (I956) qui mentionne pour la Truie des courbes ayant plusieurs maxima dans 29 p. Ioo des cas environ.

\section{Production laitière à 21 jours et production laitière totale.}

Jusqu'à trois semaines les lapereaux ne consomment que du lait maternel et leur croissance est le témoin relativement fidèle de la quantité de lait qu'ils ont reçue jusqu'alors $(r=0,96$ pour $n=\mathrm{I} 43$ ). 


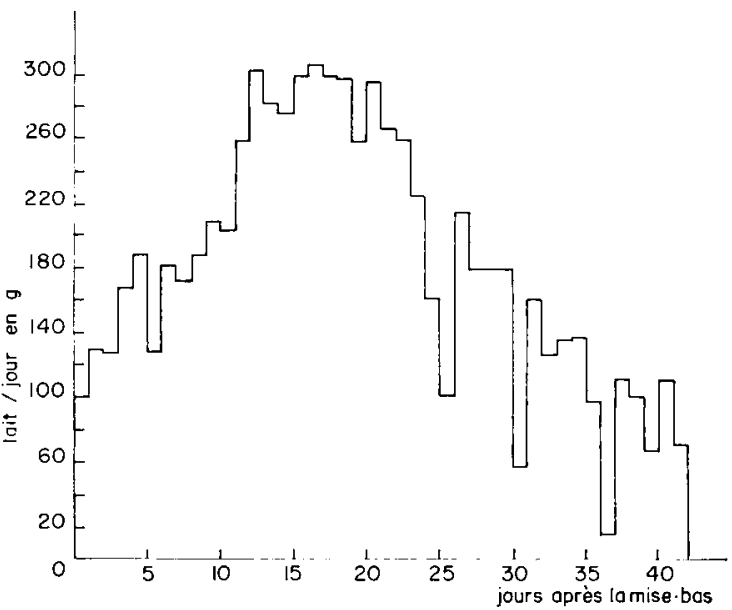

FIG. 4. - Courbe de produclion laitière de la lapine oft obtenue au cours de sa $3^{\text {e lactation }}$ (9 lapereaux élevés et sevrés) lype irrégulier

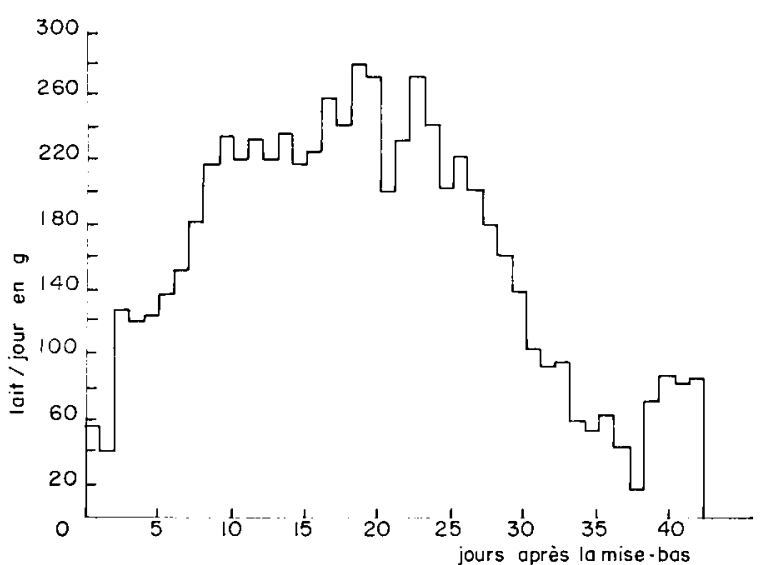

FIG. 5. - Courbe de production latière de la lapine 060 obtenue au cours de sa $2^{\mathrm{e}}$ lactation (9 lapereaux ćlevés ct sevrés) type régulier

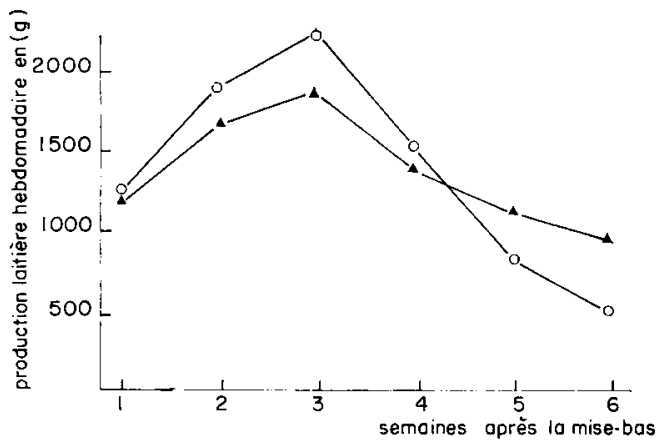

FIG. 6. - Courbes de produclion lailière de 2 lapines ayant produit des quantités globales de lait presque semblables $(8,04$ et $8,07 \mathrm{~kg})$ 
On peut utiliser cette liaison pour apprécier l'aptitude laitière des lapines, en mesurant l'augmentation de poids des lapereaux de o à $2 \mathrm{I}$ jours. Mais ceci implique que la production de lait pendant cette période soit en relation très étroite avec la quantité de lait totale produite au cours de la lactation.

Nous avons voulu vérifier cette hypothèse en calculant les coefficients de corrélation liant les différentes parties de la lactation entre elles et à la production totale. Les résultats figurent au tableau 7 .

TABLEAU 7

Relation entre les quantités de lait produites sur différentes périodes

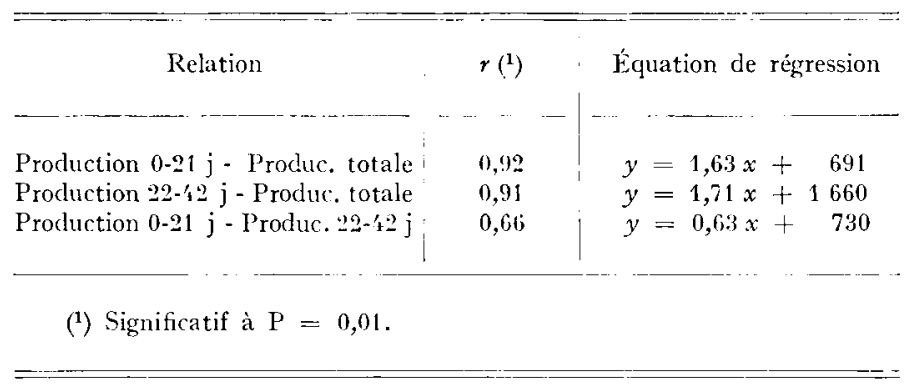

La production laitière jusqu'à 2 I jours représente plus de la moitié $(55,3 \mathrm{p}$. Ioo) de la production laitière totale des 6 semaines. La liaison étroite observée entre les deux variables justifie partiellement l'évaluation de la production laitière de la lapine d'après le gain de poids de la portée durant les trois premières semaines méthode utilisée par de nombreux éleveurs français et des auteurs comme PAvlov, (ig63) et Nigmatulin (ig63).

La relation plus faible $(r=0,66)$ entre les deux parties de la lactation est le signe d'une certaine irrégularité de forme dans les courbes de lactation.

\section{Production laitière d'une période et production laitière totale.}

Puisqu'il existe une relation étroite entre la production laitière de $O$ à 2 I jours et la production laitière totale, il est dès lors logique de rechercher s'il n'est pas possible d'évaluer la production laitière à partir d'une fraction autre que celle allant de o à 2 I jours. Les relations existant entre la production laitière totale et la production obtenue pendant des périodes plus courtes, ont été calculées. Les résultats figurent au tableau 8.

L'analyse de ce tableau montre que des coefficients de corrélation presque semblables ont été enregistrés entre la production laitière totale et des fractions de celle-ci différentes dans leur volume et leur durée. Nous avons ainsi $r=0,9$ Io pour la fraction représentant les $43,4 \mathrm{p}$. Ioo de la quantité totale de lait, obtenue en $2^{\mathrm{e}}$ et $3^{\mathrm{e}}$ semaine et $r=0,915$ pour celle totalisant 55,3 p. Ioo de cette même quantité, obtenue au cours des trois premières semaines.

Le coefficient de corrélation semble varier plus en fonction de la situation, au sein de la lactation, de la période considérée, qu'en fonction de la quantité de lait émise durant ce laps de temps. 
$\mathrm{Si}$, pour des raisons pratiques, on veut évaluer la production laitière totale à partir d'une fraction de celle-ci, pour un contrôle sur une semaine, il faut choisir la $3^{\mathrm{e}}$ ou la $4^{\mathrm{e}}$ semaine ; pour un contrôle sur deux semaines, il convient de préférer la période allant du $\mathrm{I}^{\mathrm{e}}$ au $28 \mathrm{e}$ jour de lactation ; pour un contrôle sur trois semaines, la fraction située du $8 \mathrm{e}$ au $28^{\mathrm{e}}$ jour donne les résultats les plus précis.

\section{TABLEAU 8}

Relations existant entre la production laitière totale et la production laitière durant une fraction de la lactation

$$
(n=\text { I43) }
$$

\begin{tabular}{|c|c|c|c|c|}
\hline \multicolumn{3}{|c|}{ Fraction de la lactation intéressée } & \multirow[b]{2}{*}{$r$} & \multirow{2}{*}{ Équation de régression } \\
\hline Durée & Situation & $\begin{array}{l}\text { Quantité de lait } \\
\text { p. } 100 \text { du total }\end{array}$ & & \\
\hline 1 semaine & $\begin{array}{l}1^{\text {re }} \text { semaine } \\
2^{\mathrm{e}} \text { semaine } \\
3^{\mathrm{e}} \text { semaine } \\
4^{\mathrm{e}} \text { semaine } \\
5^{\mathrm{e}} \text { semaine } \\
6^{\mathrm{e}} \text { semaine }\end{array}$ & $\begin{array}{r}11,9 \\
19,6 \\
23,8 \\
20,6 \\
14,5 \\
9,6\end{array}$ & $\begin{array}{l}0,854 \\
0,855 \\
0,884 \\
0,892 \\
0,819 \\
0,657\end{array}$ & $\begin{array}{l}y=4,740 x+3104 \\
y=4,017 x+1505 \\
y=3,587 x+1031 \\
y=3,983 x+1264 \\
y=4,126 x+2844 \\
y=3,816 x+4497\end{array}$ \\
\hline 2 semaines & $\begin{array}{l}1^{\text {re }} \text { et } 2^{\mathrm{e}} \text { semaine } \\
2^{\mathrm{e}} \text { et } 3^{\mathrm{e}} \text { semaine } \\
3^{\mathrm{e}} \text { et } 4^{\mathrm{e}} \text { semaine }\end{array}$ & $\begin{array}{l}31,5 \\
43,4 \\
44,4\end{array}$ & $\begin{array}{l}0,862 \\
0,910 \\
0,942\end{array}$ & $\begin{array}{l}y=2,537 x+1428 \\
y=2,072 x+709 \\
y=2,141 x+317\end{array}$ \\
\hline 3 semaines & $\begin{array}{lrlll}\text { de } & 0 & \text { à } & 21 & \text { jours } \\
\text { de } & 22 & \text { à } & 12 & \text { jours } \\
\text { de } & 8 & \text { à } & 28 & \text { jours }\end{array}$ & $\begin{array}{l}55,3 \\
44,7 \\
64,0\end{array}$ & $\begin{array}{l}0,915 \\
0,907 \\
0,951\end{array}$ & $\begin{array}{l}y=1,632 x+691 \\
y=1,714 x+1660 \\
y=1,509 x+240\end{array}$ \\
\hline
\end{tabular}

Pour estimer les productions laitières mentionnées ci-dessus, nous avons utilisé le contrôle des quantités de lait produites un jour sur deux. Ceci représente 2 I tétées contrôlées. Nous venons de voir qu'il existe une forte corrélation $(r=0,95 \mathrm{I})$ entre la production laitière des $2^{\mathbf{e}}+3^{\mathbf{e}}+4^{\mathbf{e}}$ semaines et la production laitière totale. Ceci représenterait également $2 \mathrm{I}$ tétées dans un contrôle quotidien.

Il semble dès lors intéressant de comparer la précision obtenue lors de l'estimation de la production laitière par deux méthodes différentes dans leur principe, mais nécessitant la même somme de travail final.

A partir des 35 lactations mesurées par un contrôle quotidien des tétées, nous avons calculé quelle aurait été l'estimation des productions laitières totales faite grâce aux équations de régression du tableau 8 pour des contrôles bloqués sur $\mathrm{I}$ ou 3 semaines, ou grâce à des contrôles régulièrement espacés sur la lactation.

Nous avons effectué ces calculs théoriques pour 7 tétées contrôlées (sur I semaine ou I fois tous les 7 jours), et pour 2 I tétées contrôlées (du $8^{\mathrm{e}}$ au $28^{\mathrm{e}}$ jour ou bien I jour sur 2). Ces résultats figurent au tableau 9.

Un certain nombre de contrôles répartis au cours de la lactation permettent une meilleure estimation que le même nombre groupé sur une seule période. Mieux, la 
même précision est obtenue avec $2 \mathrm{I}$ mesures groupées et avec 7 mesures seulement réparties sur les 42 jours. Il est donc inutile de s'astreindre à contrôler rigoureusement une partie seulement de la lactation quand un travail 3 fois moins exigeant fournit la production laitière totale avec la même précision.

TABLEAU 9

Comparaison de 4 types d'estimation de la production laitière totale obtenus par calcul à partir de 35 lactations contrôlées chaque jour, durant 6 semaines

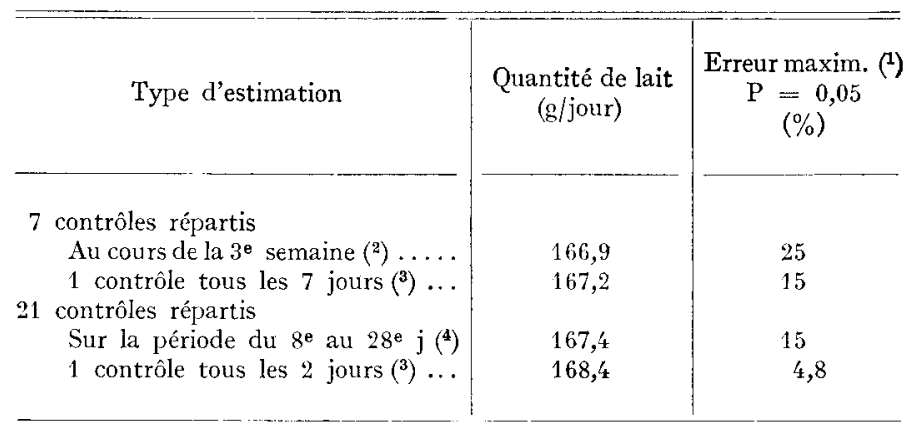

(1) Quelle que soit l'erreur, l'estimation n'est jamais significativement différente $(\mathrm{P}=0,05)$ de la mesure par contróle quotidien.

( $\left.{ }^{2}\right) y=3,587 x+1031$ et $r=0,884$.

( ${ }^{3}$ Même mode de calcul qu'au tableau 3.

(4) $y=1,509 x+2,0$ et $r=0,951$.

\section{CONCLUSIONS}

Pour étudier la lactation de la Lapine, nous avons cherché à mettre au point une méthode de mesure des quantités de lait produites, applicable à toute la lactation. Nos résultats sont de deux ordres.

\section{a) Résultats méthodologiques}

- La traite manuelle ou mécanique de la mamelle n'est pas très physiologique, elle est encore trop irrégulière dans ses techniques et ses résultats.

- Un contrôle des quantités de lait produites au cours de la tétée est possible en mesurant les variations de poids de la mère ou de sa portée pendant cette opération.

- Le contrôle par pesée de la mère plutôt que de la portée conduit à une estimation de la production laitière de la lapine avec une erreur maximum de $7,3 \mathrm{p}$. Ioo dans le cas d'un contrôle tous les deux jours.

\section{b) Contrôle de lactation}

Cent quarante-trois lactations ont été contrôlées selon cette dernière technique. Elles nous ont permis une première évaluation quantitative de la production laitière de la I,apine. 
- En 42 jours, les lapines Fauve de Bourgogne que nous avons contrôlées, ont produit en moyenne $7,09 \pm 0,28 \mathrm{~kg}$ de lait.

- La courbe de lactation est asymétrique avec un maximum situé vers I8-2 I jours après la mise bas.

- La production de la troisième semaine de lactation représente deux fois celle de la première semaine et plus de deux fois celle de la $6^{\mathrm{e}}$.

- Des variations importantes peuvent se produire dans la production laitière d'un jour à l'autre. Elles peuvent atteindre 50 à Ioo p. Ioo.

- La production laitière des 2 I premiers jours est en relation étroite avec la production totale $(r=0,92)$ mais pour les trois semaines situées du $8^{\mathrm{e}}$ au $28^{\mathrm{e}}$ jour, la relation est encore plus étroite $(r=0,95)$.

- Une estimation de la production laitière totale, calculée à partir de l'équation de régression de la production laitière de la période $8-28$ jours sur la production laitière totale, introduit une erreur maximum de $\mathrm{I}_{5} \mathrm{p}$. Ioo. L'estimation faite en contrôlant une tétée tous les 7 jours introduit la même erreur maximum de 15 p. Ioo au seuil $\mathrm{P}=0,05$.

- Dans la pratique, la technique de mesure que nous proposons est donc la suivante :

I. lorsque la précision de I5 p. IOO est estimée suffisante, il suffit de mesurer la production au cours d'une tétée tous les 7 jours par contrôle de la perte de poids de la lapine;

2. lorsqu'une précision meilleure est souhaitée, un contrôle d'une tétée sur deux dans les mêmes conditions permet l'estimation avec une erreur maximum de 7,3 p. 100 .

Reşu pour publication en mars 1968.

SUMMARY

QUANTITATIVE MEASUREMEN'T OF MILK PRODUCTION IN RABBITS

This work consists of a demonstration of a technicue for measuring the amounts of milk produced by female rabbits during the whole of the suckling period. The method proposed makes use of the estimation of loss of weight by the doe during suckling, on one day of two. This evaluation has a maximum error of 7.3 per cent in relation to the actual production.

By this method I4.3 lactations were studied. Average milk production in 6 weeks was $7.09 \pm 0.28 \mathrm{~kg}$ for the Fauve de Bourgogne breed. The mean lactation curve for 3784 individual measurements was asymetrical and showed a maximum about the 19 th day. I) ifference in production from day to day could reach 50 to roo per cent.

The relations between milk production in the whole period and certain fractions of different duration and at different times were studied. Thus, for a period of one week the closest relation was $r=0.89$ in the fourth week, for a period of 2 weeks the close'st was $r=0.94$, during the second and third weeks, and for a period of 3 weeks the correlation coefficient rise to $r=0.95$ between 8 and 28 days after parturition. In the last case estimation of total milk production by regression equation of production in 3 weeks on total production introduced a maximum error of 15 per cent at a level of significance $\mathrm{P}=0.05$. 


\section{RÉFÉRENCES BIBL,IOGRAPHIQUES}

CARrÉ P., Poly J., Vissac B., I959. Étude des méthodes de détermination des performances laitières. II. La précision d'un contrôle laitier à périodicité variable. Ann. Zoolech., 8, I I3-I 37 .

Coates M. E., Gregory M. E., 'Thompson S. Y., I964. The composition of Rabbit's milk. Brit. J. Nutr., 18, $583-586$.

Cross B. A., Harris G. W., 1952. The role of the neurohypophysis in the milk ejection reflex. J. Endocrin., 8, I 48-I6r.

Davies J. S., Widdowson I. M., McCance R. A., 1964. The intake of milk and the retention of its constituents while the newborn rabbit doubles its weight. Br. J. Nur., 18, 385-392.

Davis J., I957. Some observations on lactation and food intake of a colony of Chinchilla giganta Rabbits. J. Anim. Technicians Assoc., 7, 62-63.

Nigmatullin R. M., I963. Evaluation of milk yield of rabbits of several breeds. Krolik. Zver., 10, I3-14. Pavlov M. K., ig63. Evaluation of rabbits breeds by milk yield. Krolik. Zver., 4, 4-6.

Ricordeau G., Boccard R., Denamur R., i96o. Mesure de la production laitiere des brebis pendant la période d'allaitement. Ann. Zootech., 9, 97-г 20.

Salmon-Legagneur li., 1956. La mesure de la production laitière chez la Truie. Ann. Zootech., 5, 95-1 io. Salmon-Legagneur Ii., I958. Observations sur la production laitière des truies. Ann. Zoolech., 7, I43-162. TitareV L. A., I964. Evaluation of rabbits of various breeds by milk production. Krolik. Zver., 7 (9), 5-7, VENDT G., I93I-I932. Contribution to the theory of the nutrition of the rabbits. Utility poultry f., 17, $7^{2-75}$.

VENGE O., rg63. The influence of nursing behaviour and milk production on early growth in rabbits. Anim. Behaviour, 11, 500-506.

Zarrow M. X., Denenberg V. H., Axderson C. O., 1965. Rabbit frequency of suckling in the pup. Sci., 150, 1835-1836. 